\title{
Greyscale and contrast enhanced ultrasonography for characterization of gastric malignant tumors
}

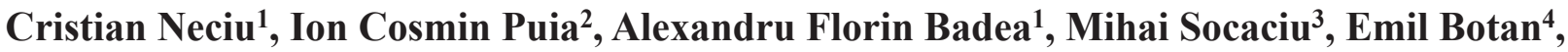 \\ Ioana Chiorean ${ }^{5}$, Iulian Opincariu ${ }^{1}$, Cornel Iancu ${ }^{2}$, Radu Badea ${ }^{6}$
}

\begin{abstract}
${ }^{1}$ Anatomy and Embryology Department, "Iuliu Hatieganu" University of Medicine and Pharmacy, ${ }^{23}$ rd Surgical Department, The Regional Institute of Gastroenterology and Hepatology "Prof. Dr. O. Fodor", "Iuliu Hatieganu" University of Medicine and Pharmacy, ${ }^{3}$ Radiology Department, The Regional Institute of Gastroenterology and Hepatology "Prof. Dr. O. Fodor", "Iuliu Hatieganu" University of Medicine and Pharmacy, ${ }^{4}$ Pathology Department, The Regional Institute of Gastroenterology and Hepatology "Prof. Dr. O. Fodor", ${ }^{5}$ Faculty of Mathematics and Informatics, BabesBolyai University, ${ }^{6}$ Ultrasonography Department, The Regional Institute of Gastroenterology and Hepatology "Prof. Dr. O. Fodor", "Iuliu Hatieganu" University of Medicine and Pharmacy, Cluj-Napoca, Romania
\end{abstract}

\begin{abstract}
Aims: To evaluate the utility of grey scale ultrasonography (US) and contrast enhanced ultrasonography (CEUS) for characterization of malignant gastric tumors. Material and methods: The study was conducted prospectively and it included a number of 30 patients with malignant gastric tumors diagnosed through upper tract endoscopy and biopsy: 25 adenocarcinomas, 3 lymphomas, and 2 stromal tumors. All the patients were examined by US, followed immediately by CEUS, using both oral and intravenous contrast agents. CEUS assessed the dynamics of the contrast agent during the arterial phase and the venous phase. The distribution characteristics of the contrast agent inside the region of interest (ROI) were also evaluated. Twenty four patients underwent surgery. Results: Adenocarcinomas presented heterogeneous enhancement with variable pattern, followed by a delayed wash-out in almost all of the situations. Lymphomas presented a variable pattern, intensity and homogeneity of the enhancement, followed by delayed wash-out. Stromal tumors showed early arterial intense and homogenous enhancement followed by moderate wash-out in venous phase. Conclusions: Grey scale US and CEUS are useful methods in characterizing gastric tumors and contribute to a more adequate evaluation of the lesions. The dynamics of the contrast agent may be suggestive for the anatomopathological nature of the tumor. Larger studies will be necessary in order to determine the practical value of the method.
\end{abstract}

Keywords: contrast enhanced ultrasonography; gastric cancer; stomach ultrasonography; tumor characterization

\section{Introduction}

The incidence of the gastric cancer patients has been decreasing during the last decades; however, the prognosis for the patients remains poor. At the moment the gastric cancer is the $5^{\text {th }}$ most frequent cancer in the world and represents the third cause of death by cancer [1].

Received 31.08.2017 Accepted 4.11.2017

Med Ultrason

2018, Vol. 20, No 1, 8-13

Corresponding author: Ion Cosmin Puia

3rd Surgical Department, Croitorilor 19-21

400613 Cluj-Napoca

E-mail: cosmin.puia@umfcluj.ro
Malignant gastric tumors are represented by adenocarcinomas $94 \%$, lymphomas $4 \%$, stromal (1\%), and other tumors $1 \%[2,3]$.

The prognosis in gastric cancer is good in early stages, stages which are usually asymptomatic. The presence of symptoms signifies local and regional advanced disease and the effectiveness of treatment is dramatically decreasing in the cases where the disease has exceeded the organ's limits .

The gold standard for diagnosis is represented by the upper tract endoscopy. This method detects tumors directly and allows a targeted biopsy, while adding endoscopic ultrasonography (US) to the exam may reveal 
parietal invasion [4-8]. In the case of ulcerative tumors, diffuse tumors, or tumors originating from deeper layers of the stomach, biopsy may be inconclusive or produces false negative results $[9,10]$.

Abdominal US is an easily available, reproducible, patient-friendly technique, which can be performed in correlation with the clinical examination. The data provided is both anatomical and functional [11,12]. The optimization of the method by using contrast agents represents a new step in improving the performance of this method. Initially used for studying vascular and parenchymal organs pathology, contrast enhanced ultrasonography (CEUS) is constantly expanding its applicability, in the last few years being used in the pathology of all abdominal organs [13].

At the moment, there are several studies regarding the preoperative diagnosis of gastric tumors using CEUS in patients with stomach cancer [14-17]. These studies are focused on highlighting the tumoral mass and less on finding a correlation between tumor histology and the behavior of the tumoral tissue on contrast administration. The vascularization of the tumoral tissue is different from that of the normal gastric wall. Tumor angiogenesis, anarchic cellular proliferation, and the development of arterial-venous shunts create a different vascular pattern from that of the normal gastric wall, thus developing the premises for a particular behavior upon vascular contrast administration [18].

The treatment of gastric tumors depends on the histology type and it is based on the wide surgical resection of the tumor and lymphadenectomy in case of the adenocarcinoma $[19,20]$, antibiotics, chemotherapy, and surgery in selected cases of gastric lymphoma [21], and in limited resection, without lymphadenectomy, accompanied by chemotherapy in stromal tumors (GIST) [22]. In this context, the preoperative recognition of the type of the disease is extremely important for the implementation of an optimal treatment.

The aim of our study was to establish the value of CEUS and grey-scale US in describing specific features of gastric tumors with respect to macroscopic appearance and histology, and thereby optimize the diagnosis of gastric cancer.

\section{Material and methods}

\section{Subjects}

The present study was conducted prospectively between 2012 and 2014, on 30 patients with gastric tumors, in 'Prof. Dr. Octavian Fodor' Gastroenterology and Hepatology Institute, Cluj-Napoca. The study was approved be local ethic committee and informed and writ- ten consent was obtained from all the patients. The inclusion criteria were: patients over 18 years old, diagnosis of a primary gastric tumor established through upper tract endoscopy and biopsy, tumor visible at transabdominal US exam. Patients with tumors originating in other structures and invading the stomach as well as cases with tumors not visible by transabdominal US exam were excluded.

\section{Ultrasonography}

The US examinations were executed by the same, highly experienced, examiner using a General Electric GE9 ultrasound machine with a multifrequency convex transducer $(2-5 \mathrm{MHz})$. All patients consumed $100 \mathrm{ml}$ oral contrast (plain water) and were examined after 30 seconds. All patients underwent standard US and CEUS examinations.

The transducer was placed in the epigastric region in two perpendicular views and several intermediate views. The vertical and horizontal parts of the stomach were examined successively. For this purpose the patients were asked to sit in a semi-sitting position. An overall visualization of the stomach and identification of the anatomic parts (cardia, vertical region, horizontal region, and pylorus) were performed. The gastric tumor was identified, localized, and characterized.

The US staging of the tumor was realized according to Bormann's classification [23]. Tumor penetration was noted as $\mathrm{u} 1=$ limited to the mucosa, $\mathrm{u} 2=$ mucosa and submucosa, $\mathrm{u} 3=$ reaching the serosa, but confined to it, and $\mathrm{u} 4=$ invasion of surrounding structures. Macroscopic form was noted as I (polypoid), II (ulcerative form with distinct, elevated borders), III (ulcerative with infiltrated base) and IV (diffuse thickening). Identification of round lymph nodes was noted $\mathrm{n} 1$, and lack of visualization $\mathrm{n} 0$. Visualization of liver metastases were noted $\mathrm{m} 1$, while the absence of metastases with $\mathrm{m} 0$. After visualizing the lesion, using the SonoLiver software, a region of interest (ROI) was drawn. This includes border ROI (including tumor and surrounding tissue), lesion ROI (perimeter of the lesion) and reference ROI (normal US scanning area located in the border ROI at $5 \mathrm{~cm}$ of lesion ROI). The blood vessels inside the ROI were evaluated by using color flow map (CFM). For this purpose the machine was set for low velocities and the image acquisition was performed with the patient in apnea. The presence of vessels was noted with 1 and their absence with 0 .

The CEUS examination immediately followed the conventional one. The contrast agent (CA) that was used was Sonovue (Bracco, Italy), in quantity of $1.6 \mathrm{~cm}^{3}$ diluted in $10 \mathrm{ml}$ saline solution. A mechanical index of 0.090.11 was used and a single focus was positioned under 
ROI. By using the "dual mode image" a combination of the fundamental and harmonic echoes was realized. The CEUS examination time was 5 minutes and the behavior of the lesion ROI was evaluated during the arterial phase (first 30 seconds from de administration of the CA) and the venous phase (the interval between 31 and $45 \mathrm{sec}$ onds from de administration).

The following US parameters were evaluated: 1) tumor enhancement with CA: present or absent; 2) the enhancement pattern of the CA during the arterial phase compared with the reference ROI: early enhancement (the tumor enhanced before the reference ROI), late enhancement (the tumor enhancement after the reference ROI), or identical enhancement of the tumor and reference ROI. The enhancement was characterized as homogeneous or inhomogeneous; 3 ) the intensity of the CA enhancement was evaluated as identical, increased, or decreased compared with reference ROI; 4) the washout pattern characterized as accelerated (when the CA left the tumor before it left the reference ROI), delayed (when the $\mathrm{CA}$ persisted in the tumor longer than the in reference ROI), or identical with that of the reference ROI. The washout phase was divided into early (the 31-45 seconds interval from the CA administration) and late washout (the 46-120 seconds interval from the CA administration). After 120 seconds from the CA administration a US evaluation of the liver was performed to highlight possible metastases.

At the end of the US examination an ultrasound staging of the tumor was performed.

\section{Surgical procedures}

Following investigations, 24 patients underwent surgery. Total or subtotal gastrectomy with eso-jejunal Roux-en-Y anastomosis or gastroduodenal anastomosis with D2 lymphadenectomy was performed. In 6 patients surgery was contraindicated.

\section{Anatomopathology}

The anatomopathologist was blinded from all preoperatory examinations. Tissue processing specimens were formalin fixed, paraffin-embedded, and hematoxylin-eosin stained. In some cases, immunohistochemical staining methods were used to ascertain the diagnose. Maximum tumor diameter, macroscopic appearance, depth invasion, histological type, degree of differentiation, and lymph node involvement were noted. The Borrmann classification of gastric cancer [23] was used to describe the macroscopic features. Adenocarcinomas were divided according to Japanese classification of gastric cancer [24]. The staging of the disease was performed according to the American Joint Committee on Cancer (AJCC) 2010 [25].

\section{Statistical analysis}

For statistical analysis we used IMB SPSS 20 statistics software. In order to determine the correlations between different variables involved in our study, we used the 2-tailed Student test. A p-value $<0.05$ was considered as statistically significant.

\section{Results}

The study was performed on 30 patients (20 male, mean age 62.5 years old). The endoscopic biopsy diagnosed 25 cases of adenocarcinoma, 3 cases of lymphoma and 2 cases of gastrointestinal stromal tumors (GIST).

There was a correlation between the enhancement pattern of CA during arterial phase and Bormann tumor type ( $\mathrm{p}=0.021$ ). Polypoid forms tend to upload CA faster, followed by ulcerative-infiltrative, ulcerative, and infiltrative forms. There was an inverse correlation between arterial uptake and tumor differentiation $(\mathrm{p}=0.021)$. For well-differentiated tumors, arterial uptake was slower than for poorly differentiated or undifferentiated tumors.

Table I. Contrast agent dynamic for adenocarcinomas, lymphomas and stromal tumors

\begin{tabular}{lllll}
\hline CA dynamics & & Types of tumors & & \\
\cline { 3 - 5 } & & Adenocarcinomas (n=25) & Lymphomas (n=3) & Stromal tumors (n=2) \\
\hline \multirow{2}{*}{ Enhancement pattern } & Early & 13 & 2 & 2 \\
& Identical & 0 & 0 & 0 \\
& Delay & 12 & 1 & 0 \\
Enhancement intensity & Intense & 4 & 3 & 2 \\
& Identical & 0 & 0 & 0 \\
Enhancement homogeneity & Weak & 11 & 0 & 0 \\
\multirow{3}{*}{ Washout pattern } & Homogenous & 4 & 0 & 0 \\
& Heterogeneous & 21 & 3 & 0 \\
& Early & 2 & 0 & 0 \\
\hline
\end{tabular}

$\mathrm{CA}=$ contrast agent; $\mathrm{n}=$ number of cases 
No correlation could be found between uptake in arterial phase and depth of invasion.

We found an inverse correlation between the maximum intensity of the capture and the degree of tumor differentiation $(p=0.024)$ (well or average differentiated tumors tend to record higher values of capture intensity compared to poorly differentiated or undifferentiated tumors) and a direct correlation between the homogeneity of the enhancement and the tumor type $(\mathrm{p}=0.034)$. GIST captures homogeneously, all lymphomas captured heterogeneously, and adenocarcinomas captured predominantly heterogeneous. No correlation between the homogeneity of CA capture and depth invasion and the degree of tumor differentiation was found. Also, there was no correlation between the mean transit time and the Bormann type, histological type, degree of tumor differentiation, and depth invasion. In Table I are de- tailed CA dynamic for adenocarcinomas, lymphomas, and GIST.

In the adenocarcinomas, the tumor behavior at CEUS examination was inhomogeneous as an expression of the differences in vascularization of these tumors (fig1). The CEUS profile of lymphomas is characterized by a variable uptake pattern, intense and heterogeneous enhancement and a delayed washout (fig 2). The profile of the studied GIST is represented by early, intense, homogeneous enhancement followed by a slow washout of the CA (fig 3).

As a result of the investigations, 24 patients underwent surgery. Out of these 24 patients, 19 were adenocarcinomas, 3 were lymphomas and 2 patients were with GIST. Grey scale US revealed liver metastases in 5 cases that were confirmed intraoperatively. In 1 patient, the surgeon found liver metastases that had not been detected by
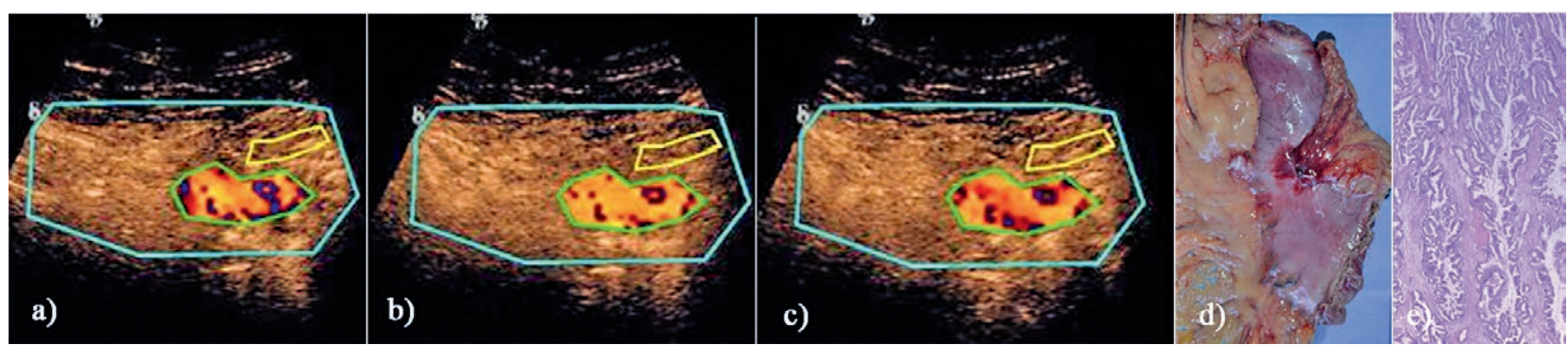

Fig 1. CEUS examination of gastric adenocarcinoma with perfusion maps showing CA uptake (a), heterogeneous enhancement (b), and delayed washout (c); d) surgery specimen of subtotal gastrectomy; e) histopathology aspect, HE 100X.
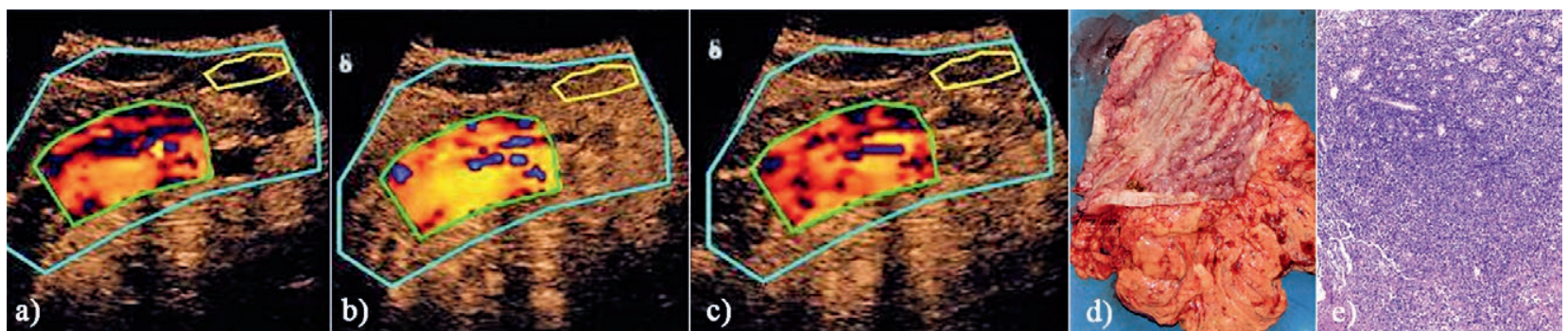

Fig 2. CEUS examination of gastric lymphoma with perfusion maps of gastric lymphoma showing intense CA uptake (a), heterogeneous enhancement (b), and delayed washout (c); d) surgery specimen of total gastrectomy; e) histopathology aspect, HE 100X.
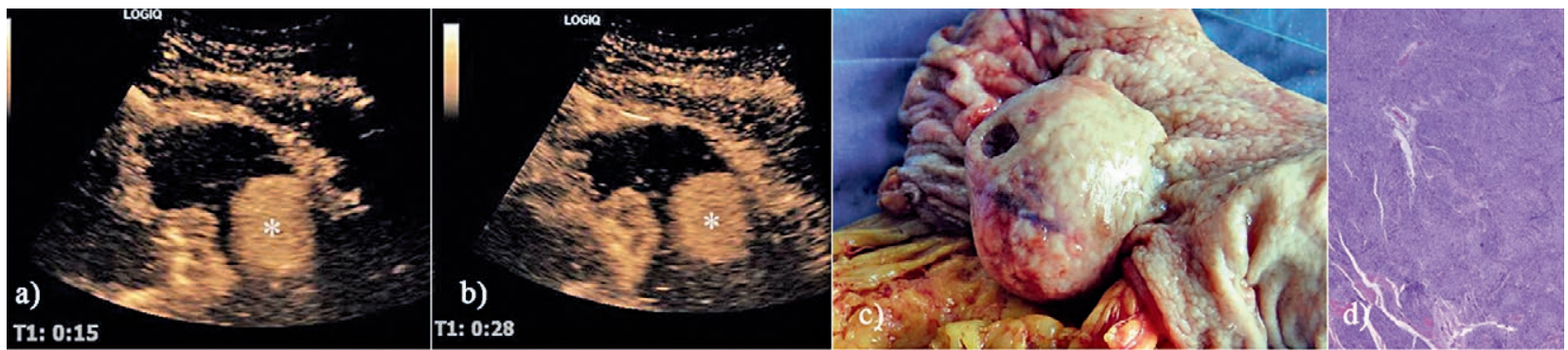

Fig 3. CEUS examination of gastric stromal tumor showing intense CA uptake, homogenous enhancement (a), and delayed washout (b); c) surgery specimen; d) histopathology aspect, HE 100X. 
Table II. Correlation between grey scale ultrasonography and pathology in tumor characterization with US accuracy

\begin{tabular}{|c|c|c|c|c|}
\hline Parameters & & $\begin{array}{l}\text { Grey-scale } \\
\text { US }\end{array}$ & Pathology & $\begin{array}{l}\text { US ac- } \\
\text { curacy }\end{array}$ \\
\hline $\begin{array}{l}\text { Macroscopic } \\
\text { form* } \\
\text { (Bormann) }\end{array}$ & $\begin{array}{l}\text { I } \\
\text { II } \\
\text { III } \\
\text { IV }\end{array}$ & $\begin{array}{l}9 \\
0 \\
7 \\
8\end{array}$ & $\begin{array}{l}9 \\
0 \\
11 \\
4\end{array}$ & $\begin{array}{l}100 \% \\
100 \% \\
63.6 \% \\
50 \%\end{array}$ \\
\hline $\begin{array}{l}\text { Tumor size } \\
(\mathrm{cm})\end{array}$ & $\begin{array}{l}<2 \\
2<4 \\
4<6 \\
6<8 \\
8<10 \\
>10\end{array}$ & $\begin{array}{l}2 \\
3 \\
6 \\
3 \\
5 \\
5\end{array}$ & $\begin{array}{l}2 \\
3 \\
7 \\
2 \\
3 \\
7\end{array}$ & $87.5 \%$ \\
\hline Depth invasion & $\begin{array}{l}\text { u1/T1 } \\
\text { u2/T2 } \\
\text { u3/T3 } \\
\text { u4/T4 }\end{array}$ & $\begin{array}{l}1 \\
5 \\
13 \\
5\end{array}$ & $\begin{array}{l}2 \\
1 \\
10 \\
11\end{array}$ & $58.3 \%$ \\
\hline Lymph nodes & $\begin{array}{l}\text { n0 } \\
\text { n1 }\end{array}$ & & $\begin{array}{l}6 \\
18\end{array}$ & $91.6 \%$ \\
\hline $\begin{array}{l}\text { Liver metas- } \\
\text { tases }\end{array}$ & $\begin{array}{l}\mathrm{m} 0 \\
\mathrm{~m} 1\end{array}$ & $\begin{array}{l}19 \\
5\end{array}$ & $\begin{array}{l}18 \\
6\end{array}$ & $95.5 \%$ \\
\hline Stage & $\begin{array}{l}1 \\
2 \\
3 \\
4\end{array}$ & $\begin{array}{l}1 \\
6 \\
11 \\
6\end{array}$ & $\begin{array}{l}1 \\
3 \\
12 \\
8\end{array}$ & $87.5 \%$ \\
\hline
\end{tabular}

*I polypoid, II ulcerative form with distinct, elevated borders, III ulcerative with infiltrated base, IV diffuse thickening

US. The grey scale US and anatomopathology findings in the surgical patients are listed in Table II.

\section{Discussions}

Literature studies demonstrate a high utility of ultrasonography in the diagnosis of symptomatic gastric cancer [26]. The development of the CEUS created the premises for characterizing the tissue microcirculation [27]. Being highly sensitive in detecting pathological alterations of the microcirculation [28-30], it allows not only to identify the tumoral tissue compared to normal gastric wall but also to differentiate between adenocarcinoma, lymphoma, and GIST. Gastric adenocarcinomas are both macroscopically and microscopically heterogeneous tumors, and this characteristic explains the variable pattern of CEUS which consists of accelerated or delayed enhancement, predominantly heterogeneous, followed by a delayed CA washout. These features are consistent with the feeding vessels and distorted nourishing vasculature of gastric carcinoma described by Shi et al [31]. We could not find published data about the CEUS profile of gastric lymphomas, but in our cases the tumor was characterized by a variable uptake pattern, intense and heterogeneous enhancement, and a delayed washout. Concerning GIST, we found an early, intense, homogeneous enhancement followed by a slow washout of the CA, the same as Shi et al [31] and Stock et al [32] .

US and CEUS allowed us a good appraisal of Borrmann macroscopic types, tumor size, lymph node involvement, and a fair measurement of depth invasion, consistent with the results obtained by Fang Wei et al [33]. A step forward may have been the use of CEUS in following the response to neoadjuvant chemotherapy before surgical resection in patients with locally-advanced gastric cancer [34].

The main weakness of the study is the number of patients enrolled in the study, which gives the study limited accuracy, especially in the case of GIST and lymphomas. Although blinded to the endoscopic and histopathologic results, the ultrasonographer was aware of the presence of a gastric tumor. No intra- or interobserver variability was investigated. No case of linitis plastica was present in our study. Six of our patients were not eligible for surgery and in these cases no correlations could be made between the US appearance and the macroscopic tumor characteristics and the anatomopathological staging.

\section{Conclusions}

US combined with CEUS is a promising imaging technique for the macroscopic characterization of gastric tumors and for giving a hint on the histological type in cases where biopsy is inconclusive or negative and thus influence therapy. We proved the value of CEUS by combining qualitative and semiquantitative evaluations in the assessment of the various histopathological tumor types.

US and CEUS does not substitute upper tract endoscopy, but it brings additional data about tumor macroscopic type, size, penetration, and lymph node involvement. Further studies are necessary in order to define the accuracy and indications of the method.

\section{Conflict of interests: none}

\section{References}

1. GLOBOCAN 2012: Estimated cancer incidence, mortality and prevalence worldwide in 2012. Available at: http://globocan.iarc.fr/Pages/fact_sheets_cancer.aspx

2. Severson RK, Davis S. Increasing incidence of primary gastric lymphoma. Cancer 1990;66:1283-1287.

3. deKorwin JD. Epidemiology of Helicobacter pylori infection and gastric cancer. Rev Prat 2014;64:189-193.

4. Mickevicius A, Ignatavicius P, Markelis R, et al. Trends and results in treatment of gastric cancer over last two decades 
at single East European centre: a cohort study. BMC Surg 2014;14:98.

5. Koch P, Probst A, Berdel WE, et al. Treatment results in localized primary gastric lymphoma: data of patients registered within the German multicenter study (GIT NHL 02/96). J Clin Oncol 2005;23:7050-7059.

6. DeMatteo RP, Lewis JJ, Leung D, Mudan SS, Woodruff JM, Brennan MF. Two hundred gastrointestinal stromal tumors: recurrence patterns and prognostic factors for survival. Ann Surg 2000;231:51-58.

7. Matsumoto Y, Yanai H, Tokiyama H, Nishiaki M, Higaki S, Okita K. Endoscopic ultrasonography for diagnosis of submucosal invasion in early gastric cancer. J Gastroenterol 2000;35:326-331.

8. Tsendsuren T, Jun SM, Mian XH. Usefulness of endoscopic ultrasonography in preoperative TNM staging of gastric cancer. World J Gastroenterol 2006;12:43-47.

9. Andriani A, Zullo A, Di Raimondo F, et al. Clinical and endoscopic presentation of primary gastric lymphoma: a multicentre study. Aliment Pharmacol Ther 2006;23:721726.

10. Scherübl H, Faiss S, Knoefel WT, Wardelmann E. Management of early asymptomatic gastrointestinal stromal tumors of the stomach. World J Gastrointest Endosc 2014;6:266271.

11. Haruma K, Kusunoki H, Manabe N, et al. Real-time assessment of gastroduodenal motility by ultrasonography. Digestion 2008;77 Suppl 1:48-51.

12. Gilja $\mathrm{OH}$, Hatlebakk JG, Odegaard S, et al. Advanced imaging and visualization in gastrointestinal disorders. World J Gastroenterol 2007;13:1408-1421.

13. Chung YE, Kim KW. Contrast-enhanced ultrasonography: advance and current status in abdominal imaging. Ultrasonography 2015;34:3-18.

14. Wang J, Yang Y, Ding L, et al. Diagnostic value of contrastenhanced ultrasonography in preoperative Borrmann classification of gastric cancer. Zhonghua Wei Chang Wai Ke Za Zhi 2014;17:254-257.

15. Wang CL, Yang YM, Cui J, et al. Diagnostic value of double contrast-enhanced ultrasonography in preoperative staging of gastric cancer. Zhonghua Zhong Liu Za Zhi 2009;31:701-704.

16. Wei F, Huang P, Li S, et al. Enhancement patterns of gastric carcinoma on contrast-enhanced ultrasonography: relationship with clinicopathological features. PLoS One 2013;8:e73050.

17. Zheng Z, Yu Y, Lu M, et al. Double contrast-enhanced ultrasonography for the preoperative evaluation of gastric cancer: a comparison to endoscopic ultrasonography with respect to histopathology. Am J Surg 2011;202:605-611.

18. Badea AF, Tamas-Szora A, Clichici S, et al. Contrast enhanced ultrasonography (CEUS) in the characterization of tumor microcirculation. Validation of the procedure in the animal experimental model. Med Ultrason 2013;15:85-94.
19. Ito H, Clancy TE, Osteen RT, et al. Adenocarcinoma of the gastric cardia: what is the optimal surgical approach? J Am Coll Surg 2004;199:880-886.

20. Jiang L, Yang KH, Chen Y, et al. Systematic review and meta-analysis of the effectiveness and safety of extended lymphadenectomy in patients with resectable gastric cancer. Br J Surg 2014; 101:595-604.

21. Kato M, Asaka M. Recent knowledge of the relationship between Helicobacter pylori and gastric cancer and recent progress of gastroendoscopic diagnosis and treatment for gastric cancer. Jpn J Clin Oncol 2010;40:828-837.

22. Kim IH, Kim IH, Kwak SG, Kim SW, Chae HD. Gastrointestinal stromal tumors (GISTs) of the stomach: a multicenter, retrospective study of curatively resected gastric GISTs. Ann Surg Treat Res 2014;87:298-303.

23. Borrmann R. Geschwülste des Magens. In: Henke F, Lubarsch O (eds.). Handbuch der speziellen pathologischen Anatomie und Histologie, Band IV/1, Springer: Berlin, Heidelberg, New York; 1926.

24. Japanese Gastric Cancer Association. Japanese classification of gastric carcinoma: 2nd English edition. Gastric Cancer 1998:10-24.

25. Stomach. In: Edge SB, Byrd DR, Compton CC, et al. (eds.). AJCC Cancer Staging Manual. 7th ed. New York, NY: Springer, 2010:117-126.

26. Martinez-Ares D, Aguirre PA, López Y, et al. Sensitivity of ultrasonography for gastric cancer diagnosis in symptomatic patients. Dig Dis Sci 2009;54:1257-1264.

27. Cosgrove D, Lassau N. Imaging of perfusion using ultrasound. Eur J Nucl Med Mol Imaging 2010;37:S65-S85.

28. Quaia E. Microbubble ultrasound contrast agents: an update. Eur Radiol 2007; 17:1995-2008.

29. Correas JM, Bridal L, Lesavre A, Méjean A, Claudon M, Hélénon O. Ultrasound contrast agents: properties, principles of action, tolerance, and artifacts. Eur Radiol 2001;11:1316-1328.

30. Nagy JA, Chang SH, Dvorak AM, Dvorak HF. Why are tumour blood vessels abnormal and why is it important to know? Br J Cancer 2009;100:865-869.

31. Shi H, Yu XH, Guo XZ, et al. Double contrast-enhanced two-dimensional and three-dimensional ultrasonography for evaluation of gastric lesions. World J Gastroenterol 2012;18:4136-4144.

32. Stock K, Hann von Weyhern C, Slotta-Huspenina J, et al. Microcirculation of subepithelial gastric tumors using contrast-enhanced ultrasound. Clin Hemorheol Microcirc 2010;45:225-232.

33. Wei F, Huang P, Li S, et al. Enhancement patterns of gastric carcinoma on contrast-enhanced ultrasonography: relationship with clinicopathological features. PLoS One 2013;8:e73050.

34. Ang J, Hu L, Huang PT, et al. Contrast-enhanced ultrasonography assessment of gastric cancer response to neoadjuvant chemotherapy. World J Gastroenterol 2012;18:70267032 . 